\title{
NOVEDADES BIBLIOGRÁFICAS
}

New Books

2016

\author{
NOVEDADES EN CASTELLANO
}

\section{Traducciones}

Así habló Zaratustra, trad. de A. Sánchez Pascual, Madrid: Alianza, 560 pp. ISBN: $978-$ 84-9104-254-9.

El nacimiento de la tragedia, trad. de J. B. Llinares, Madrid: Tecnos, 256 pp. ISBN: 978 84-3096-897-8.

La Gaya Ciencia, trad. de J. L. Vermal Madrid: Tecnos, 336 pp. ISBN: 978-84-3096895-4.

Obras Completas IV. Escritos de Madurez II y Complementos a la Edición, ed. dirigida por Sánchez Meca, Madrid: Tecnos, 1192 pp. ISBN: 978-84-7702-812-3.

Obras Completas I. Escritos de Juventud, ed. dirigida por D. Sánchez Meca, Madrid: Tecnos, 984 pp. ISBN: 978-84-3096-880-0.

\section{Monografías}

Hollingdale, R. J., Nietzsche. El hombre y su filosofía, trad. de C. García Trevijano, y M. Garrido Giménez, Madrid: Tecnos, 336 pp. ISBN: 978-84-3096-879-4.

Medrano, J. M., El reino Celestial del Cambio, Madrid: Bibilioteca Nueva, 440 pp. ISBN: 978-84-1664-770-5.

Mora Mínguez, T., Eterno retorno, narración y autosuperación: Las raíces antropológicas del pensamiento de Nietzsche, Madrid: Brumaria, 400 pp. ISBN: 978-84-9440059-9.

Rivero Weber, P. (ed.), Nietzsche: El desafío del pensamiento, México: Fondo de Cultura Económica, 326 pp. ISBN: 978-60-7163-530-3.

\section{NOVEDADES EN INGLÉS}

Berger, K., Beyond Reason: Wagner contra Nietzsche, California: University of California Press.

Bishop, P., On the Blissful Islands with Nietzsche and Jung: In the shadow of the Superman, London: Routledge. 
Blue, D., The Making of Friedrich Nietzsche: The Quest of Identity, 1844-1869, Cambridge: University of Cambridge Press.

Bowman, W., Friedrich Nietzsche: Herald of a New Era, Illinois: Hazar Press.

Conard, Mark, Nietzsche and the Philosophers (Routledge Studies in Nineteenth Century Philosophy),

D'Lorio P., Nietzsche's Journey to Sorrento: Genesis of the Philosophy of the Free Spirit, Chicago: University of Chicago Press.

Deane, D., Nietzsche and Theology: Nietzschean Thought in Christological Anthropology (Ashgate New Critical Thinking in Religion, Theology and Bible), London: Routledge.

Daw Nay, R. y Evans R., Nietzsche and Classical Greek Philosophy: Beautiful and Diseased, Maryland: Lexington Books.

Drochon, H., Nietzsche's Great Politics, Princeton: Princeton University Press.

Jensen, A. K., An Interpretation of Nietzsche's «On the Uses and Disavantages of History for Life», London: Routledge.

Fuentes, C., Nietzsche on His Balcony, Illinois: Dalkey Archive Press.

Hunt, L. H., Nietzsche of the Origin of Virtue, London: Routledge.

Russell, J., Nietzsche and the Clinic: Psychoanalysis, Phylosophy, Metaphysics, London: Karnac Books.

Schrift, A., Nietzsche and the Question of Interpretation, London: Routledge.

Shapiro, G., Nietzsche's Earth: Great Events, Great Politics, Chicago: University of Chicago Press.

\section{NOVEDADES EN FRANCÉS}

Bouveresee, J., Nietzsche contre Foucault: Sur le vérité, la connaissance et le pouvior, Marseille: Agone.

Denat, C., Wotling, P., Nietzsche: Les premiers textes sur les Grecs, Reims: Presses Universitaires Reims.

Merker, A., Nietzsche: Philosophie et philologue, Strasburg: Presses Universitaires de Strasbourg.

Monia, S., L'Inconscient politique chez Friedrich Nietzsche, Saint-Denis: Connaissances et savoirs.

Tonning, G., Nietzsche: Una Philosophie de l'Épreuve, Paris: Ellipses Marketing.

\section{NOVEDADES EN ALEMÁN}

Bürger, P., 'Nach vorwärts erinnern': Relektüren zwischen Hegel und Nietzsche, Göttingen: Wallstein.

Celestini, F., Nietzsches Musikphilosophie, Paderborn: Wilhelm Fink.

Ferraris, M., Nietzsches Gespenster: Ein menschliches und intellektuelles Abentener, Frankfurt: Vittorio Klostermann.

Friedrich, O., Aurenque, A., Assadi, G., Schleiden, S., Nietzsche, Foucault und die Medizin: Philosophische Impulse für die Medizinethik (Bioethik / Medizinethik), Bielefeld: transcript.

Georg, J., Reschke, R., Nietzsche und Wagner: Perspektiven ihrer Auseinandersetzung, Berlin: De Gruyter.

Gödde, G., Loukidelis, N., Zirfas, J., Nietzsche und die Lebenskunst: Ein pilosophischpsychologisches Kompendium, Stuttgart: J. B. Metzler.

Günther, M., Hitler und Nietzsche. Oder wie ein Philosoph doch noch Geschichte machte: Eine kriminalsoziologische Studie, Kappelrodeck: Deutscher Wissenschafts-Verlag. 
Heit, H. y Thorgeirsdottir, S. (eds.), Nietzsche als Kritier un Denker der Transformation, Berlin: De Gruyter.

Kunnas, T., Politik als Prostitution des Geistes: Eine Studie über das Politische bei Nietzsche, Rohrdorf: Briena Verlag.

Scheinbenberg, S., Historischer und kritischer Kommentar zu Friedrich Nietzsches Werken: Kommentar zu Nietzsches «Ueber Wahrheit und Lüge im aussermoralischen Sinne, Berlin: De Gruyter.

Schmidt, J., Der Mythos «Wille zur Macht»: Nietzsches Gesamtwerk und der Nietzsche-Kult. Eine historische Kritik, Berlin: De Gruyter.

Sonneberg, S.-M., Nietzsche nackt, Linz am Rhein, Linz: Verlag 3.0.

Summer, A. U., Historischer und kritischer Kommentar Zu Friedrich Nietzsches Werken: Kommentar zu Nietzsches «Jeinseits voc Gut und Böse», Berlin: De Gruyter.

Wellbery, D. E., Traum und Rausch bei Nietzsche und Svevo, Konstanz: UVK Verlagsgesellschaft.

Ziegler, T., Die. F.N.- Schlaufe. Ernstes und Heiteres aus dem Leben des fabelheaften Friedrich Nietzsche: Ein Bilderbuch, Naumburg: Stiftung.

Zudeick, P., Nietzsche für Eilige, Berlin: Aufbau Taschenbuch.

\section{NOVEDADES EN ITALIANO}

Amato, P., Politica e tragedia. La filosofia del giovane Nietzsche, Genova: Il nuevo Melangolo.

Belviso, F., Amor fati. Pavese all'ombra di Nietzsche, Torino: Aragno.

Caffo, L., Del destino dell'uomo. Nietzsche e i quattro errori dell'umanità, Prato: Piano B.

Carteny, V., La giustizia di Dionisio. Nietzsche: l'oltre-uomo, il legislatore, Roma: Bonanno.

Crudi, L., Dell'aristofanesca irrisione del mondo. Nietzsche, al secolo XIX, Torino: Robin.

D’Alessandro, P., Lo stile del pensiero. Una lettura ipertestuale del caso emblemático di Friedrich Nietzsche, Milano: LED Edizioni Universitarie.

De Pourtalès, G., Nietzsche in Italia, Cesena: Historica Edizione.

Evola, J., Par dela' Nietzsche, Torino: Aragno.

Fergnani, F., Nietzsche e la filosofia dell'esistenza, Milano: Farina Editore.

Gori, P., Il pragmatismo di Nietzsche. Saggi sul pensiero prospettivistico, Sesto San Giovanni: Mimesis.

Nuzzo, V., Il grande nemico della tradizione, Forlì: Victrix.

Olivieri, R., Nietzsche tra le stelle. L'eterno ritorno e la cosmologia, Saonara: Il Prato.

Papparo, C. F., Qualcosa del corpo. Nietzsche e la scena dell'anima, Pisa: Edizioni ETS.

Penzo, G., Invito al pensiero di Friedrich Nietzsche, Milano: Ugo Mursia Editore.

Zacchini, S., Una instable armonia. Gil anni della giovinezza di Friedrich Nietzsche, Pisa: Edizioni ETS.

Zavatta, L., Il diritto nella volontà di potenza. Saggi su Nietzsche, Ariccia: Aracne.

Antonio Rovi Ruiz

Universidad de Málaga 